\title{
An impoverished machine: Challenges to human learning and instructional technology
}

\author{
ROMAN TARABAN \\ Texas Tech University, Lubbock, Texas
}

\begin{abstract}
Many of the limitations to human learning and processing identified by cognitive psychologists over the last 50 years still hold true, including computational constraints, low learning rates, and unreliable processing. Instructional technology can be used in classrooms and in other learning contexts to address these limitations to learning. However, creating technological innovations is not enough. As part of psychological science, the development and assessment of instructional systems should be guided by theories and practices within the discipline. The technology we develop should become an object of research like other phenomena that are studied. In the present article, I present an informal account of my own work in assessing instructional technology for engineering thermodynamics to show not only the benefits, but also the limitations, in studying the technology we create. I conclude by considering several ways of advancing the development of instructional technology within the SCiP community, including interdisciplinary research and envisioning learning contexts that differ radically from traditional learning focused on lectures and testing.
\end{abstract}

Every society has a history, and the Society for Computers in Psychology (SCiP) is no exception. ${ }^{1}$ The first meeting was held in 1971, under the name the National Conference on the Use of On-Line Computers in Psychology (NCUOLCP). It was not until 1982 that the present name was adopted (Sidowski, 1990). A survey of early proceedings reveals numerous contributions that consisted largely of electronic schematics or computer code and that were no more than a few pages in length. SCiP's mission as a forum and a source of information on how to build and code computers and other devices for the purpose of psychological research continues to the present day, as a cursory review of recent conference papers readily reveals. Beyond the terse reports focused on building a circuit (Overton \& Overton, 2007) or coding a routine (Wright, 2007), the work of the Society encompasses cognitive modeling using computers (see, e.g., Burgess, 1998; Tzeng, van den Broek, Kendeou, \& Lee, 2005), statistical routines (e.g., Sheu, Chen, Su, \& Wang, 2005), and online privacy issues (Proctor \& Vu, 2007). It also includes the development, testing, and dissemination of software utilities for tasks such as constructing experiments and collecting data (Schneider, Zuccolotto, \& Eschman, 2007), computing statistical power (Faul, Erdfelder, Lang, \& Buchner, 2006), collecting experimental data over the World-Wide Web (Reips, 2006), providing clinical consultations at a distance (Wade \& Wolfe, 2004), coding observational data (MacLin \& MacLin, 2005), analyzing eye movements (Myers \& Schoelles, 2005), and formatting documents in APA style (Li \& Cunningham, 2005).

An emerging area of interest has to do with computers and learning in applied settings, and it covers topics related to observational training (Ray \& Ray, 2006), tutoring in content areas (e.g., Graesser, 2003), assessing reading strategies (Gilliam, Magliano, Millis, Levinstein, \& Boonthum, 2007), teaching reading strategies (Levinstein, Boonthum, Pillarisetti, Bell, \& McNamara, 2007), teaching psychology (Bradshaw, Steinman, \& McCarley, 2002), and the assessment of instructional software (e.g., Maki \& Maki, 2001; Taraban, Maki, \& Rynearson, 1999; Taraban, Rynearson, \& Stalcup, 2001). Using computers for instruction has been an interest of mine, and it is the topic on which I will focus in the remainder of this article.

The theme of my presidential address is the impoverished machine. This label derives in large measure from information-processing psychology, which applied the metaphor of the mind as a computer to human cognition. On the basis of several decades of research on limitations to human learning, it may be more realistic to think of the human agent as an impoverished machine. Practically, our knowledge of constraints on human computation provides approximate benchmarks for applying technology to learning. Beyond these rough benchmarks, the technology we develop needs to be an object of our research, like any other phenomena we study within psychological science. By thinking about the methodology that we use to develop

R. Taraban, roman.taraban@ttu.edu 
technological innovations for instruction and learning, we can derive additional criteria for assessing technological products. Specifically, the methods we apply in developing and assessing instructional technology should be productive, and our conclusions should be empirically supported (Proctor, 2005; Proctor \& Capaldi, 2006). I present an informal account of my own work in assessing instructional technology for engineering thermodynamics to show not only the benefits, but also the limitations, in studying the technology we create. I conclude with some suggestions for expanding the Society's involvement in the development of instructional technology.

\section{The Impoverished Machine:}

\section{Human Computation and Knowledge}

The computational theory of mind (Aaronson, 1994; Massaro, 1986; Thagard, 2005) has dominated cognitive psychology since its reemergence in the 1950s. At a functional level, it doesn't matter much what substrate you choose for computation. The essential computations can be carried out on a variety of materials - for example, vacuum tubes, neurons, or silicon chips (Simon, 1990). What matters is what gets computed, and much of what we understand today in developmental theory (Siegler \& Alibali, 2005) or adult cognition (Anderson, 2007) is couched in terms of representation and process - the twin elements of computation.

Since its reemergence in the late 1950s, the study of cognition has been involved in a beneficial partnership with computer science and, particularly, with artificial intelligence research (Aaronson, 1994). As our knowledge of human and artificial intelligence has advanced over the years, there have been a number of scholarly comparisons of human and machine intelligence (e.g., Nakamura, Taraban, \& Medin, 1993). However, it is also possible to ask a more practical question and inquire about how human intelligence compares with machine intelligence, as one benchmark of the power of human computation. Recent history gives some hints. On February 10, 1996, a computer system named "Deep Blue" won a chess game against reigning world chess champion, Garry Kasparov, under regular game conditions. Deep Blue played Kasparov again in May of 1997 and won a six-game match under standard tournament conditions. Thus, a machine was able to win against a world-champion chess player, confirming (albeit a bit late) a prediction made by Herbert Simon in 1957 that within 10 years, a computer would be able to beat the world's top chess player. IBM promoted the idea that Deep Blue's playing strength was derived from brute computing force (Simon \& Munakata, 1997). The 1997 machine was capable of evaluating 200 million positions per second (Hsu, 2002) - a search speed well beyond human capability. In contrast, Simon and Munakata suggested that the victory was primarily due to Deep Blue's superior chess knowledge - particularly chess patterns - and selective search. Specifically, Simon and Munakata attributed the computer's victory to the same knowledge base and search abilities that underlie human intelligence. On either account, the machine beat Kasparov at mental computation.

As a further challenge to human cognition, information storage appears to be low. In a 1986 article, Tom Landauer attempted to estimate the quantity of learned information in long-term memory. He considered the rate at which individuals input information (through reading and looking at pictures) and estimated it at $2 \mathrm{bits} / \mathrm{sec}$. He also used available research on memory loss to come up with an estimate for information loss of $10^{-9} \mathrm{bits} / \mathrm{bit} / \mathrm{sec}$. This yielded a net gain over 70 years of $1.4 \times 10^{9}$ bits. Dividing by 8 gives $175 \mathrm{MB}$ of memory, which is hardly enough to fill a common computer CD. Chi and Ohlsson (2005) used Landauer's estimate to speculate that humans accumulate about a million pieces of knowledge over a lifetime. The brain may have a lot of capacity-estimated at between $10^{12}$ and $10^{14}$ synapses (Landauer, 1986) - but what is relevant is how much information is actually stored. On this point, Landauer used the analogy of shelf space in a library versus the number of books on the shelves - that is, lots of shelf space, but not many books. It is also possible that the brain is inefficient in its use of storage capacity and codes information with "massive redundancy" (Landauer, 1986, p. 492) and "profligate use of capacity" (p. 493), so that much of the brain's capacity is used, but not very effectively.

A firm assessment of the state of human knowledge and learning can be drawn from behavioral research. For humans, it is easy to forget, distort, and misattribute (Schacter, 1999), to hold false memories and to be manipulated by questions (Loftus, 1997). It is now well known and accepted in cognitive research that humans are limited-capacity computational systems. The notion of a limited-capacity system is revealed in psychological principles, like bounded rationality (Simon, 1990); in the properties of working memory systems (Morrison, 2005); in empirical findings, like those related to the so-called seven sins of memory (Schacter, 1999); heuristic judgment (Kahneman \& Frederick, 2005), and to the 10-year rule for developing expertise in domain (Ericsson, Krampe, \& Tesch-Römer, 1993; Hayes, 1981).

Human learning does not come easy. The speculative work of Landauer (1986) and others - as well as empirical results like those cited - provide a sense of the computational constraints on learning and performance and associated costs in "upgrading" human thinking. Technology has been applied to learning for quite some time-for example, in mainframe systems like Plato, which was developed at the University of Illinois in the 1970s - and software for personal computers, like the popular Oregon Trail used at the elementary school level to teach planning and decision making in the 1980s. Applications within this Society include AutoTutor (Graesser, 2003), which tutors students in topics such as physics using a natural language interface. When technology is applied to learning and instruction, the constraints on human learning can be used to set approximate expectations for learning outcomes and performance. These expectations can be adjusted on the basis of usability studies and other analyses of students' performances that target the goals of the software-for example, learning information, or developing critical thinking ability. In a bolder fashion, one might try to estimate the time required to master a set of instructional modules or a course of study prior to implementing it, using Landauer's estimate of $2 \mathrm{bits} / \mathrm{sec}$ as a starting point for knowledge gained. Although this suggestion may seem far-fetched, a contemporary movie titled 
Two Million Minutes (Compton \& Heeter, 2007) indirectly does something like that. The film documents how high school students in the United States, China, and India spend the 2 million minutes that constitute the time they spend in high school and considers the educational ramifications of differences in study times in the three countries.

\section{Instructional Technology \\ Coupled With Methodology}

Technology can be used to develop learning applications. But technology by itself is not enough. Robert Proctor, in his 2004 Presidential Address to SCiP, emphasized the need to incorporate scientific methodology into the development of technology (Proctor, 2005). Basically, one needs an effective way of thinking about the technology one is developing, and work must be grounded in scientific theory and practice. For Proctor, that meant immersing oneself in the core beliefs and empirical approaches in psychology, as well as seeking ways of deciding on the adequacy of empirical and theoretical claims. Proctor and Capaldi (2006) elaborated on this idea, advocating that we submit the methodology we use in our research to the same scrutiny, testing, and evaluation that we give to the psychological phenomena of interest: Methodology itself should be an object of science.

Methodology comes into play at several levels. Pedagogical theories about how students learn (see, e.g., Bransford, Brown, \& Cocking, 2000) and how they learn through specific forms of technology, like multimedia learning (e.g., Mayer, 2001), are applied through methodological choices related to the design of the software. Separately, research methodology is used to assess the impact of the technology on the learner. In the more fundamental sense of methodology used in Proctor and Capaldi (2006), it becomes an object of study. These three aspects of methodology — and perhaps others - are relevant to developing technology, but seldom are all aspects considered. Generally, researchers focus on results, not on why specific methodologies were chosen, nor on how effective they were in achieving the intended goals for the product. I present an informal account of my own work in assessing instructional technology for engineering thermodynamics and reflect on the methodology using two criteria from the work of Proctor (2005; Proctor \& Capaldi, 2006): The methods we apply in developing and assessing instructional technology should be productive, and our conclusions must be justified empirically.

The research in my example was conducted with a colleague in mechanical engineering, Ed Anderson. Anderson had authored supplemental software for undergraduate thermodynamics. The software was designed to elicit active learning by including auditory, verbal, and visual inputs, and by regularly requiring the learner to interact with the materials. There were screens with text; screens that combined text, figures, and graphics; screens requiring student interaction - for example, controlling simulations (see Figure 1) - and quiz screens (see Figure 2). Each screen began with a spoken narration related to the content on the particular screen. We set a goal of evaluating its effectiveness in helping students to learn.

The theoretical orientation that we adopted was related to the notion of an active learner. Active learners set goals during learning; they apply strategies in order to build coherent knowledge structures; they revise misconceptions; they monitor their performance; and they generally take control of their own learning (Bransford et al., 2000). We applied experimental methods in an opportunistic manner, choosing methods that seemed appropriate as the assessment progressed.

One step involved determining how students distributed their study times across available learning resources, which included the Anderson CD (Taraban, Hayes, Anderson, \& Sharma, 2004). The participants were students enrolled in introductory thermodynamics at two universities, and data were collected for three semesters. Log dataself-recorded by students over the course of a weeksuggested that students did not often use the instructional CD (see Figure 3, "Use CD-ROM"). Rather, they most frequently attended class and solved problems from the

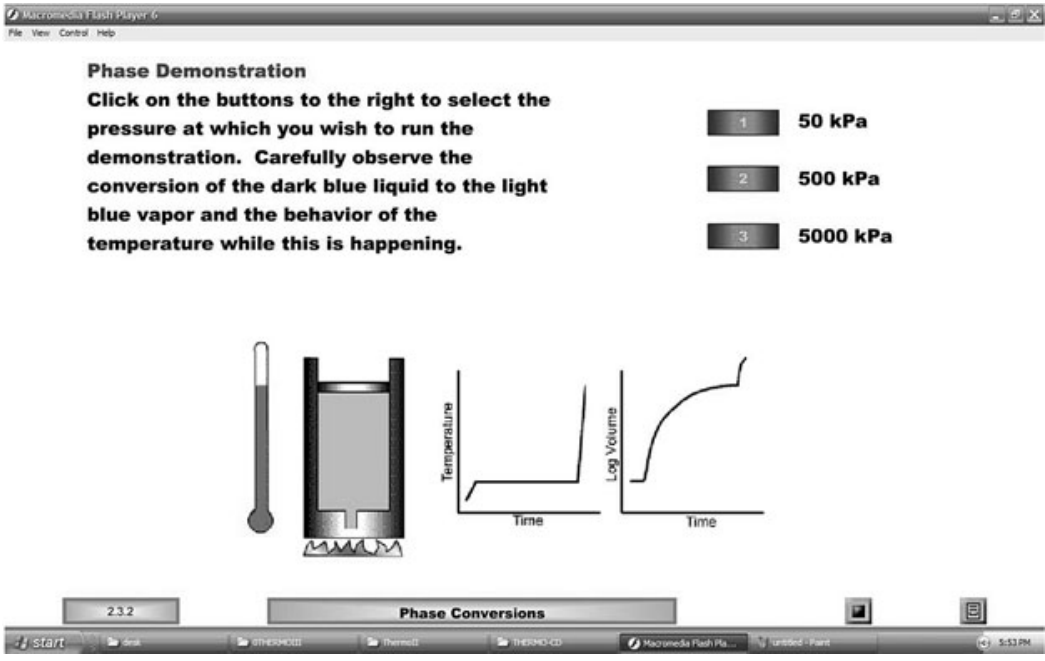

Figure 1. Sample interactive screen from the thermodynamics software. 

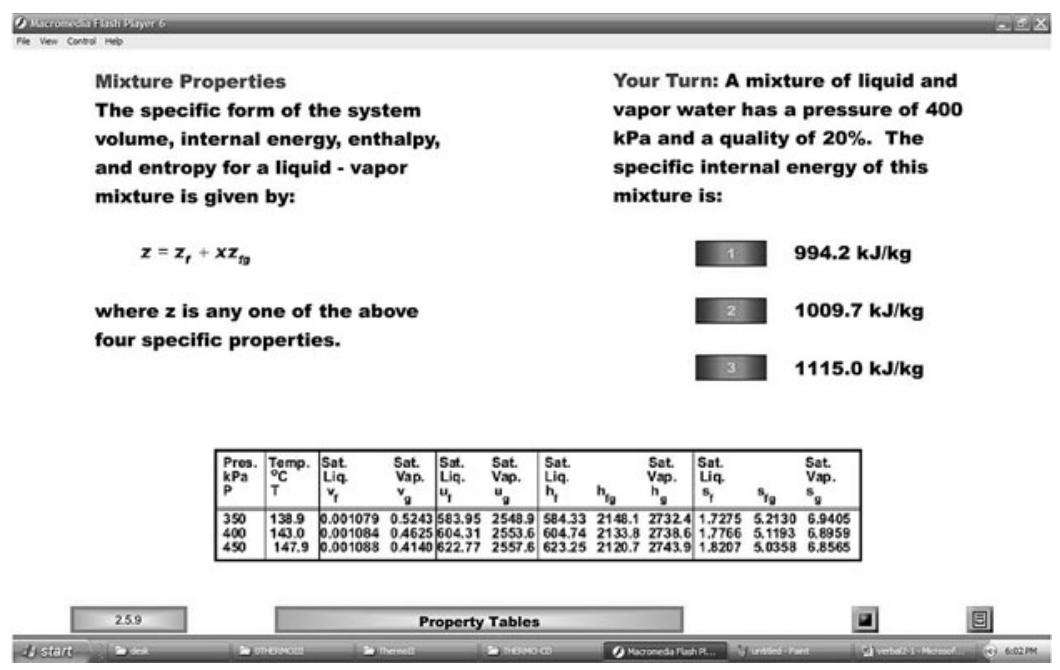

Figure 2. Sample quiz screen from the thermodynamics software.

textbook. From these data, it appeared that students valued other learning resources over the instructional $\mathrm{CD}$, and we could expect that, overall, the CD would have little impact on class performance measured through standard instruments such as in-class tests. This was indeed the case. Students used the CD less than $30 \mathrm{~min} /$ week, on average, and time spent on the $\mathrm{CD}$ did not correlate significantly with students' homework grades, in-class test grades, final grades, or the grade they expected to get for the course.

To better understand students' motivations and cognitions when using the $\mathrm{CD}$, we reasoned that simple pro- cess tracing could reveal something about what students were thinking as they progressed through the computer screens. The logic came from research on metacognitive processing in reading (Pressley, Brown, El-Dinary, \& Afflerbach, 1995; Wyatt et al., 1993), which suggested that skilled individuals are active while reading, posing questions, making inferences, jumping forward and backward in the text, and responding emotionally to the author. Process traces collected from students who used the software in regular courses revealed a predominant pattern of moving forward to the next computer screen

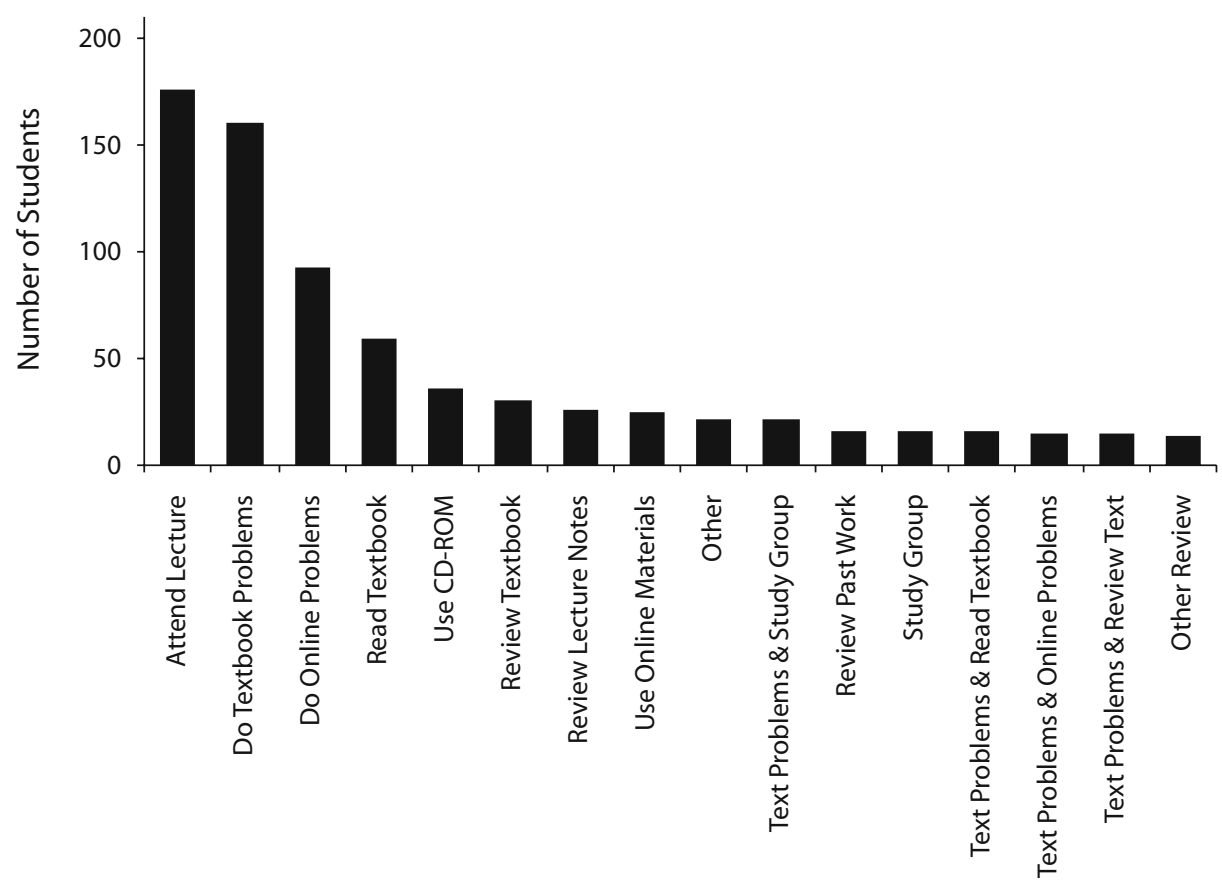

Learning Resources

Figure 3. Number of students reporting use of learning resources in thermodynamics courses at the University of Wyoming and Texas Tech University $(N=211)$ in a typical week of instruction. 
(Taraban, Anderson, Sharma, \& Weigold, 2003). This pattern typically accounted for over $90 \%$ of the transitions in the data samples we collected (see Figure 4 for an example). There were essentially none of the screen navigation behaviors that would have suggested an active and metacognitive learner.

The two sets of data suggested that the software was not achieving the intended learning goals. However, because process tracing could not provide specific information about cognitive processing, it was still unclear what students were doing while using the software. Therefore, we turned to one of the most direct methods of eliciting cognitions and collected verbal protocol data as students worked through the software (Ericsson \& Simon, 1993; Pressley $\&$ Afflerbach, 1995). The first experiment that we conducted involved science and engineering majors who were appropriate candidates for the thermodynamics course, but who had not taken it. In a subsequent experiment, we collected data from a sample of students who were enrolled in the thermodynamics course (Thermo I), as well as from students who had completed the course and were taking the next course in thermodynamics (Thermo II). All participants worked through the same modules in the software, which corresponded to the early chapters in the course textbook. The codes that we developed for these data distinguished between lower and higher level cognitive processes. Examples of lower level processes are (1) reads or repeats verbatim, (2) shows early comprehension (e.g., pulling out words from text, providing rudimentary description of graph), and (3) paraphrases (i.e., summarizes somewhat coherently). Examples of higher level cognitions are: (1) makes inference about content, (2) connects information in current screen, (3) connects to previous screens, (4) connects to outside knowledge, (5) explains, (6) draws conclusion, and (7) applies comprehension strategy (e.g., "I'll wait and see"; "I'll retry the simulation"). Two significant findings emerged (Taraban, Definis, Brown, Anderson, \& Sharma, 2007):

1. The format of the computer screens mattered. Students generally did not engage in higher level cognitive processes. However, to the extent that they did, computer screens requiring interactions were more likely to evoke these.

2. In comparing pre-Thermo, Thermo I, and Thermo II students, there were no significant differences in the frequency of lower versus higher level cognitions. However, in comparing students who received a lower course grade ( $B$ or below) with students who received a higher course grade ( $A$ students) across the Thermo I and Thermo II samples, there was a significant interaction between course grades and lower and higher level cognitions. Specifically, higher grade students applied fewer lower level cognitions than did lower grade students. Higher grade students also engaged in more higher level cognitions than did lower grade students.

From these data, we concluded that interactive learning contexts are relatively more evocative of higher level cognitive processes than text-oriented contexts are, that learners do not necessarily process information more deeply as they advance through the engineering curriculum, and that academically successful students (on the basis of thermodynamics course grades) process information at higher cognitive levels than do less successful students.

What about our methodology? First, conclusions were supported empirically, fulfilling one of the criteria from Proctor (2005; Proctor \& Capaldi, 2006). This claim, however, needs to be qualified in all the standard ways: There is a need to replicate the findings, there needs to be better control over sampling (particularly minimizing participant self-selection), and conclusions could be tested more rigorously by converting some aspects of the studies into controlled experiments. On a positive side, participants in the first two studies were drawn from two universities,



Figure 4. Total number of moves (and \%) from current page in the thermodynamics software for a sample of University of Wyoming students $(n=44)$. 
and data were collected over several semesters, thereby eliminating some potential confounds and providing more generality to the findings.

Regarding the second criterion, the methods were productive in tentatively disconfirming our expectations that students would use the software readily (on the basis of the $\log$ data) and that they would use it in an active manner (on the basis of the process-trace data). These two experimental formats - that is, questionnaire and automatic process stamping by the computer - were chosen on the basis of their simplicity in implementation and coding. Had we stopped there and not implemented the more time-consuming verbal protocol studies, we would have missed key findings about the software. One was that students were more cognitively active (and perhaps more motivated) on screens requiring interactions. Another was that the software had limited utility. Students who were already academically successful (highgrade students) worked through the software with more frequent higher level cognitions than did less academically successful students; students who needed most to develop critical thinking skills were exercising those skills the least.

In sum, the research methodologies that we used were weak, falling short of Proctor's ideal of "clean experimental designs" (Proctor, 2005, p. 198). However, they yielded several findings that could lead to deeper insights into when and how the technology works and when it does not. The present example treated the methods as an object of science in an informal manner, but was helpful by increasing awareness of the costs and benefits of the methods, as well as by considering their validity and appropriateness for the empirical and theoretical questions being asked.

Proctor's (2005; Proctor \& Capaldi, 2006) general admonition is to ground technology in the theories and practices of psychological science. Although I have provided reasons for supporting that position throughout this section, at times it may be more productive to simply look at the learning task from the perspective of a student. What is the student's experience with the technology like, and how can the technology be changed to give students the experiences that will produce learning (Simon, 1998)? There are many questions that arise in the research I described; for example, was active learning an appropriate theory to use to design the software; were the computational constraints of the learner sufficiently acknowledged; and, should motivation have been given more attention? One can try to respond to these formally by appealing to alternative theories. However, as was suggested by Simon (1998), there may be times when it is more productive to approach questions like these heuristically by analyzing the tasks and taking the students' perspectives.

In retrospect, discovering and applying the appropriate methodologies used in this research example was a bit of a puzzle that required drawing on appropriate theory, devising and evaluating effective experimental methods, and, to some degree, muddling through messy real-life contexts subject to multiple experimental biases and confounds. Advice from Proctor (2005) to focus on methodology is well taken. Without the guidance afforded by a good theoretical base and knowledge of not only how to exploit, but also how to evaluate, experimental methods, one risks produc- ing technological innovations without deeper insights into the phenomena that are being addressed. Our technological innovations will succeed to the extent that we pay more deliberate attention to methodology at multiple levels.

\section{Leveraging SCiP Resources to Advance the Development of Instructional Technology}

On a practical note, what are some ways in which to support and expand research on instructional technology within SCiP? A committee of the National Academies (National Academy of Sciences, 2005) recently suggested that interdisciplinary research is productive and inspiring, and that, through conversations and cross-disciplinary connections, interdisciplinary research leads to deeper understanding, new knowledge, and new technologies. Danielle McNamara (2006), in an editorial piece for the journal Cognitive Science, picked up a similar theme and advocated interdisciplinary research, particularly between cognitive scientists and educators. As McNamara pointed out, as researchers, our knowledge of perception, language, and cognition has matured, so we are as ready as ever to apply that knowledge to real-world problems. Practically speaking, she noted that government and private agencies are funding interdisciplinary ventures. By drawing on opportunities to share expertise across disciplinary boundaries, Society members can reap the benefits highlighted by the National Academies, contribute to raising the quality of formal education, and possibly gain financial support for their research.

Participating in interdisciplinary research is one way of leveraging resources within $\mathrm{SCiP}$ to further develop instructional technology. A second way is to envision our research in alternative contexts. In a broad survey of the effectiveness of undergraduate research programs - like those funded through the National Science Foundation's Research Experiences for Undergraduates program and the Howard Hughes Medical Institute's Science Education program - my colleague in plant biology, Richard L. Blanton, and I confirmed that research experiences benefit students by helping them to develop lab skills, an appreciation of the scientific method, and peer and faculty relations (Taraban \& Blanton, 2008). To find an effective role for computers and technology in a reformed curriculum (e.g., one in which undergraduates spend more time in authentic research settings) would lead in different directions than trying to develop tools and technologies in current educational settings (typical lecture and test venues). Thus, we need to pay attention to the contexts in which we carry out our work. There should be room for "what if" questions in our research - for example, what if folks took the current call for science curriculum reform seriously; what kind of software tools would be most effective? Being creative in envisioning new learning contexts and new learning technologies is a second way to leverage $\mathrm{SCiP}$ resources.

Finally, our work should contribute no less to theory building and testing than that of our colleagues doing basic research. The research reviewed earlier involving thermodynamics software indicated that learning contexts requiring active student involvement are more evocative of higher level cognitions than of more passive contexts. This finding provides a basis for exploring a more general 
learning principle that holds true with or without the use of instructional technology. Adding to basic theoretical knowledge related to learning is a third way in which $\mathrm{SCiP}$ can leverage resources.

\section{Conclusions}

In this address, I have touched on three guiding principles for the development of instructional technologies:

1. There are inherent constraints on human computation and the acquisition and retention of knowledge. Simon (1990) regarded many of these constraints as law-like invariants of human cognition. These constraints should be recognized when developing instructional technology and used to set expectations for learning outcomes.

2. The technological innovations that we develop should be an object of research, like the other phenomena we study within psychological science. The methods we apply in developing and assessing instructional technology should be productive, and our conclusions should be supported empirically (Proctor, 2005; Proctor \& Capaldi, 2006).

3 . Interdisciplinary collaborations and research must grow within the Society in order to keep our work relevant in the 21 st century; we need to envision revolutionary contexts in which our research has meaning and credibility, and we should contribute to the learning theories in our domains. Envisioning new opportunities and radical revisions to current practices is consistent with scientific methodology properly understood (Proctor, 2005; Proctor \& Capaldi, 2006).

Failing to acknowledge constraints on the user and the need to rigorously and systematically design and test the products we develop will inevitably lead to an empty use of technology. Using my own experiences as a way to "connect the dots" in this article, it is apparent that "clean experimental designs" (Proctor, 2005, p. 198) are hard to achieve in applied settings. In the lab, we can calibrate the task to the participant to avoid floor effects and ceiling effects; in the real world, we may be surprised to find that the learner knows less (or more) than we anticipated. Treating technology as an object of research in the most fundamental sense (Proctor, 2005; Proctor \& Capaldi, 2006) is also not easily achieved; however, it does provide a promising channel through which to advance the impact of technological innovation.

In conclusion, the primary purpose of our Society is to increase and diffuse knowledge of the use of computers in psychological research. Members of the Society have developed some of the leading tools for Internet research (e.g., Reips, 2006), lab research (e.g., Schneider et al., 2007), and statistical analyses (e.g., Faul et al., 2006; Sheu et al., 2005). High-capacity and high-speed computers allow the exploration of high-dimensional semantic spaces for basic and applied research projects (Westbury, 2007). The powerful interfacing of basic research in language comprehension-combined with sophisticated technology - has produced instructional engines like AutoTutor, which was the topic of a keynote address by Art Graesser at the 2003 SCiP Conference (Graesser, 2003). In yet other research combining basic theories of human cognition with fMRI imaging technology, John Anderson
(2003) is bridging mind and brain, with practical implications for learning and instruction. He is yet another one of the keynote speakers who has benefited the Society by sharing his work with us. This sampling of articles and presentations shows that the Society is succeeding in its mission. Sidowski (1990), in his historical account of the Society some 20 years ago, noted that "perhaps there was little need to continue [the Society], since "everyone knows about computers now"' (p. 96). I don't think so.

This presidential address has given me an opportunity to acknowledge some of the excellent work that has been completed by Society members over the years. My several years on the steering committee and as president have been important to me personally and professionally, and, in parting, I would simply like to say thank you.

\section{AUTHOR NOTE}

This work was supported in part by a grant from the Howard Hughes Medical Institute Undergraduate Science Education Program to Texas Tech University. I thank Curtis Craig for comments on an earlier version of the manuscript. Correspondence concerning this article should be addressed to R. Taraban, Department of Psychology, Texas Tech University, Lubbock, TX 79409 (e-mail: roman.taraban@ttu.edu).

\section{REFERENCES}

Aaronson, D. (1994). Computer use in cognitive psychology. Behavior Research Methods, Instruments, \& Computers, 26, 81-93.

Anderson, J. R. (2003, November). How should a theory of learning and cognition inform instruction? Keynote address presented at the 33rd Annual Meeting of the Society for Computers in Psychology, Vancouver, BC.

Anderson, J. R. (2007). How can the human mind occur in the physical universe? Oxford: Oxford University Press.

Bradshaw, G. L., Steinman, B., \& McCarley, N. (2002). ePsych: Interactive demonstrations and experiments in psychology. Behavior Research Methods, Instruments, \& Computers, 34, 231-233.

Bransford, J. D., Brown, A. L., \& Cocking, R. R. (EDs.) (2000). How people learn: Brain, mind, experience, and school. Washington, DC: National Academies Press.

BuRGESs, C. (1998). From simple associations to the building blocks of language: Modeling meaning in memory with the HAL model. Behavior Research Methods, Instruments, \& Computers, 30, 188-198.

Castellan, N. J., JR. (1991). Computers and computing in psychology: Twenty years of progress and still a bright future. Behavior Research Methods, Instruments, \& Computers, 23, 106-108.

ChI, M. T. H., \& OHLsson, S. (2005). Complex declarative learning. In K. J. Holyoak \& R. G. Morrison (Eds.), The Cambridge handbook of thinking and reasoning (pp. 371-399). New York: Cambridge University Press.

Compton, R. A. (Executive Producer), \& Heeter, C. (Director) (2007). Two million minutes: A documentary film on global education [Motion picture]. United States: Broken Pencil Productions.

Ericsson, K. A., Krampe, R. T., \& Tesch-Römer, C. (1993). The role of deliberate practice in the acquisition of expert performance. Psychological Review, 100, 363-406.

Ericsson, K. A., \& Simon, H. A. (1993). Protocol analysis: Verbal reports as data (Rev. ed.). Cambridge, MA: MIT Press.

FAul, F., Erdfelder, E., LANG, A., \& Buchner, A. (2006, November). $G^{*} P O W E R$ 3: A statistical power analysis program for $t-, F-, z_{-}$, $\chi^{2}-$ and exact tests in the social, behavioral, and biomedical sciences. Paper presented at the 36th Annual Meeting of the Society for Computers in Psychology, Houston, TX.

Gilliam, S., Magliano, J. P., Millis, K. K., Levinstein, I., \& Boonthum, C. (2007). Assessing the format of the presentation of text in developing a Reading Strategy Assessment Tool (R-SAT). Behavior Research Methods, 39, 199-204.

GraEsser, A. C. (2003, November). Evolution of computer learning environments: From computer assisted instruction to intelligent tutoring 
systems with natural language dialogue. Keynote address presented at the 33rd Annual Meeting of the Society for Computers in Psychology, Vancouver, BC

Hayes, J. R. (1981). The complete problem solver. Philadelphia: Franklin Institute Press.

Hsu, F.-H. (2002). Behind Deep Blue: Building the computer that defeated the world chess champion. Princeton, NJ: Princeton University Press.

Kahneman, D., \& Frederick, S. (2005). A model of heuristic judgment. In K. J. Holyoak \& R. G. Morrison (Eds.), The Cambridge handbook of thinking and reasoning (pp. 267-293). New York: Cambridge University Press.

LANDAUER, T. K. (1986). How much do people remember? Some estimates of the quantity of learned information in long-term memory. Cognitive Science, 10, 477-493.

Levinstein, I. B., Boonthum, C., Pillarisetti, S. P., Bell, C., \& McNamaRA, D. S. (2007). iSTART 2: Improvements for efficiency and effectiveness. Behavior Research Methods, 39, 224-232.

Li, P., \& Cunningham, K. (2005). The APA Style Converter: A Webbased interface for converting articles to APA style for publication. Behavior Research Methods, 37, 219-223.

LofTus, E. F. (1997). Memories for a past that never was. Current Directions in Psychological Science, 6, 60-65.

MacLin, O. H., \& MacLin, M. K. (2005). Coding observational data: A software solution. Behavior Research Methods, 37, 224-231.

MAKI, W. S., \& MAKI, R. H. (2001). Mastery quizzes on the Web: Results from a Web-based introductory psychology course. Behavior Research Methods, Instruments, \& Computers, 33, 212-216.

Massaro, D. W. (1986). The computer as a metaphor for psychological inquiry: Considerations and recommendations. Behavior Research Methods, Instruments, \& Computers, 18, 73-92.

MAYER, R. E. (2001). Multimedia learning. Cambridge: Cambridge University Press.

MCNAmaRA, D. S. (2006). Bringing cognitive science into education, and back again: The value of interdisciplinary research. Cognitive Science, 30, 605-608.

Morrison, R. G. (2005). Thinking in working memory. In K. J. Holyoak \& R. G. Morrison (Eds.), The Cambridge handbook of thinking and reasoning (pp. 457-473). New York: Cambridge University Press.

Myers, C. W., \& Schoelles, M. J. (2005). ProtoMatch: A tool for analyzing high-density, sequential eye gaze and cursor protocols. Behavior Research Methods, 37, 256-270.

Nakamura, G. V., Taraban, R., \& Medin, D. L. (Eds.) (1993). The psychology of learning and motivation: Vol. 29. Categorization by humans and machines. San Diego: Academic Press.

National Academy of Sciences (2005). Facilitating interdisciplinary research. Washington, DC: National Academies Press.

OVERTON, R. L., \& OVERTON, D. A. (2007). A high-sensitivity drinkometer circuit with 60-Hz filtering. Behavior Research Methods, 39, 118-122.

Pressley, M., \& AfFLERBACH, P. (1995). Verbal protocols of reading: The nature of constructively responsive reading. Hillsdale, NJ: Erlbaum.

Pressley, M., Brown, R., El-Dinary, P. B., \& Afflerbach, P. (1995). The comprehension instruction that students need: Instruction fostering constructively responsive reading. Learning Disabilities Research \& Practice, 10, 215-224

Proctor, R. W. (2005). Methodology is more than research design and technology. Behavior Research Methods, 37, 197-201.

Proctor, R. W., \& CAPALDI, E. J. (2006). Why science matters: Understanding the methods of psychological research. Malden, MA: Blackwell.

Proctor, R. W., \& Vu, K.-P. L. (2007). A multimethod approach to examining usability of Web privacy policies and user agents for specifying privacy preferences. Behavior Research Methods, 39, 205-211.

Ray, R., \& RaY, J. (Co-Chairs) (2006, November). Digital video and artificially intelligent multimodal expert systems: Training and teaching applications in psychology. Symposium presented at the 36th Annual Meeting of the Society for Computers in Psychology, Houston, TX.

REIPS, U.-D. (2006, November). Internet-based psychological research: See more. Do more. Presidential address presented at the 36th Annual Meeting of the Society for Computers in Psychology, Houston, TX.

SCHACTER, D. L. (1999). The seven sins of memory: Insights from psychology and cognitive neuroscience. American Psychologist, 54, 182-203.

Schneider, W., Zuccolotto, A., \& Eschman, A. (Co-Chairs) (2007,
November). E-Prime 2.0 Professional: Improving the power, precision, and ease of use of a proven research standard. Symposium presented at the 37th Annual Meeting of the Society for Computers in Psychology, Long Beach, CA.

Sheu, C.-F., Chen, C.-T., SU, Y.-H., \& WANG, W.-C. (2005). Using SAS PROC NLMIXED to fit item response theory models. Behavior Research Methods, 37, 202-218.

SidowsKi, J. B. (1990). Past, present, and future of the Society for Computers in Psychology. Behavior Research Methods, Instruments, \& Computers, 22, 94-97.

Siegler, R. S., \& Alibali, M. W. (2005). Children's thinking (4th ed.). Upper Saddle River, NJ: Prentice Hall.

Simon, H. A. (1990). Invariants of human behavior. Annual Review of Psychology, 41, 1-19.

Simon, H. A. (1998). What we know about learning. Journal of Engineering Education, 87, 343-348.

Simon, H. A., \& Munakata, T. (1997). AI lessons. Communications of the $A C M, 40,23-25$.

Taraban, R., Anderson, E. E., Sharma, M. P., \& Weigold, A. (2003, June). Developing a model of students' navigations in computer modules for introductory thermodynamics. Paper presented at the American Society for Engineering Education Annual Conference \& Exposition, Nashville, TN.

Taraban, R., \& Blanton, R. L. (EDs.) (2008). Creating effective undergraduate research programs in science: The transformation from student to scientist. New York: Teachers College Press.

Taraban, R., Definis, A., Brown, A., Anderson, E. E., \& Sharma, M. P. (2007). A paradigm for assessing conceptual and procedural knowledge in engineering students. Journal of Engineering Education, 96, 335-345.

Taraban, R., Hayes, M. W., Anderson, E. E., \& Sharma, M. P. (2004). Giving students time for the academic resources that work. Journal of Engineering Education, 93, 205-210.

Taraban, R., Maki, W. S., \& Rynearson, K. (1999). Measuring study time distributions: Implications for designing computer-based courses. Behavior Research Methods, Instruments, \& Computers, 31, 263-269.

Taraban, R., Rynearson, K., \& Stalcup, K. A. (2001). Time as a variable in learning on the World-Wide Web. Behavior Research Methods, Instruments, \& Computers, 33, 217-225.

Thagard, P. (2005). Representation and computation. In Mind: Introduction to cognitive science (2nd ed., pp. 3-22). Cambridge, MA: MIT Press.

Tzeng, Y., van den Broek, P., Kendeou, P., \& Lee, C. (2005). The computational implementation of the landscape model: Modeling inferential processes and memory representations of text comprehension. Behavior Research Methods, 37, 277-286.

Wade, S. L., \& Wolfe, C. (2004, November). Putting the pieces together: Testing the efficacy of a Web-based intervention for families of children with traumatic brain injury. Paper presented at the 34th Annual Meeting of the Society for Computers in Psychology, Minneapolis, $\mathrm{MN}$.

Westbury, C. (Chair) (2007, November). Co-occurrence and lexical organization. Symposium presented at the 37th Annual Meeting of the Society for Computers in Psychology, Long Beach, CA.

Wolfe, C. R. (2006). SCiP at 35: An idiosyncratic history of the Society for Computers in Psychology. Behavior Research Methods, 38, $245-250$

WRIGHT, D. B. (2007). Graphing within-subjects confidence intervals using SPSS and S-Plus. Behavior Research Methods, 39, 82-85.

Wyatt, D., Pressley, M., El-Dinary, P., Stein, S., Evans, P., \& Brown, R. (1993). Comprehension strategies, worth and credibility monitoring, and evaluations: Cold and hot cognition when experts read professional articles that are important to them. Learning \& Individual Differences, 5, 49-72.

\section{NOTE}

1. For excellent reviews of SCiP's past, see Sidowski (1990), Castellan (1991), and Wolfe (2006).

(Manuscript received December 6, 2007; revision accepted for publication March 25, 2008.) 